\title{
Percepción de la pandemia COVID-19 en los servicios de cirugía en Colombia
}

\author{
Perception of the COVID-19 pandemic in the departments of surgery in Colombia
}

\author{
Carlos E. Díaz-Castrillón ${ }^{1}$, Natalia Cortés ${ }^{1}$, Samuel Rey ${ }^{1}$, Manuela Pineda², \\ Juan F. Díaz-Castrillón ${ }^{3}$, Sara Sierra ${ }^{4}$

\begin{abstract}
Médico, Especialista en Cirugía General, Pontificia Universidad Javeriana, Bogotá DC., Colombia
Médico, Residente de Cirugía General, Pontificia Universidad Javeriana, Bogotá DC., Colombia

Médico General, Universidad del Bosque, Bogotá DC., Colombia
\end{abstract} \\ Médico, Especialista en Anestesiología, Pontificia Universidad Javeriana, Bogotá DC., Colombia
}

\section{Resumen}

Introducción. La pandemia por COVID-I9 ha llevado a modificar la estructura asistencial en los hospitales alrededor del mundo, buscando la mejor forma de adaptarse para responder al incremento de pacientes infectados por el virus, sin desantender las necesidades de los pacientes no afectados por el mismo. El objetivo de este trabajo es describir la percepción de la comunidad quirúrgica sobre el impacto que ha tenido la pandemia COVID-I9 en los servicios de cirugía en Colombia.

Métodos. Estudio de corte transversal descriptivo mediante encuesta estructurada compuesta por io preguntas de selección múltiple y 8 preguntas con items tipo Likert. Se distribuyó electrónicamente a residentes de cirugía general, cirujanos generales o sub-especialistas que tuvieran práctica clínica en Colombia.

Resultados. Se incluyeron 464 participantes, $27.8 \%$ fueron mujeres y $45.3 \%$ cirujanos generales. Alrededor de la mitad residían en Bogotá (5I.4 \%) o trabajaban en un hospital universitario privado (48.3 \%). El 78.8\% de los encuestados consideraron que las recomendaciones de seguridad son efectivas y $76.6 \%$ se sienten seguros realizando procedimientos con ellas. Sin embargo, el 63\% consideran que estas se cumplen parcialmente. Hasta el momento, $20 \%$ de los participantes reportó haber tenido contacto directo con pacientes COVID-I9 positivos. Finalmente, la mayoría (93\%) estan de acuerdo con la cancelación de procedimientos electivos y estan dispuestos a ser reubicados en otros servicios en caso de ser necesario.

Conclusiones. Para el inicio de la fase de mitigación en Colombia existe consenso en la percepción del impacto de la pandemia COVID-ı9 en los servicios de cirugía. No se observó una variabilidad significativa respecto al nivel de entrenamiento ni al tipo de institución hospitalaria.

Palabras claves: COVID-I9; Virus del SRAS; coronavirus; infecciones por coronavirus; pandemias; percepción; encuestas y cuestionarios.

Fecha de recibido: 30/04/2020 - Fecha de aceptación: 01/05/2020

Autor de correspondencia: Carlos Eduardo Díaz-Castrillón, M.D.

Faculty Pavilion-Fifth Floor, 4401 Penn Avenue, Pittsburgh, PA 15224. Telefono: (+1) 412-580-3204

Correo electrónico: cediazc@gmail.com

Citar como: Díaz-Castrillón CE, Cortés N, Rey S, Pineda M, Díaz-Castrillón JF, Sierra S. Percepción de la pandemia COVID-19 en los servicios de cirugía en Colombia. Rev Colomb Cir. 2020;35:290-301. https://doi.org/10.30944/20117582.655

Este es un artículo de acceso abierto bajo una Licencia Creative Commons - BY-NC-ND https://creativecommons.org/licenses/by-ncnd/4.0/deed.es 


\begin{abstract}
Introduction: The COVID-I9 pandemic has led to changes in the care structure in hospitals around the world, seeking the best way to adapt in response to the increase in patients infected with the virus, without neglecting the needs of patients not affected by it. The objective of this study is to describe the perception of the surgical community on the impact that the COVID-I9 pandemic has had on the surgical services in Colombia. Methods: Descriptive cross-sectional study using a structured survey consisting of ro multiple-choice questions and 8 questions with Likert-type items. It was distributed electronically to residents of general surgery, general surgeons, or sub-specialists who had clinical practice in Colombia.
\end{abstract}

Results: 464 participants were included, $27.8 \%$ were women and $45.3 \%$ were general surgeons. About half resided in Bogotá $(5 \mathrm{I} .4 \%$ ) or worked in a private university hospital (48.3\%). $78.8 \%$ of the respondents considered that the safety recommendations are effective and $76.6 \%$ feel safe performing procedures with them. However, $63 \%$ consider that these are partially met. So far, $20 \%$ of participants reported having had direct contact with COVID-I9 positive patients. Finally, the majority (93\%) agree to the cancellation of elective procedures and are willing to be relocated to other services if necessary.

Conclusions: There is a consensus in the perception of the COVID-I9 pandemic impact in the surgery services without observing significant variability regarding the level of training or the type of hospital institution.

Key words: COVID-I9; SARS Virus; coronavirus; coronavirus infections; pandemics; perception; surveys and questionnaires.

\section{Introducción}

El establecimiento de la pandemia por SARSCoV-2 ha llevado a afrontar grandes desafios, tanto en el ambito laboral como en el personal. Desde el punto de vista de la comunidad médica, la misión es propender por hacer lo mejor posible para responder a las necesidades de los sistemas de salud, y el grupo de médicos pertenecientes al área quírurgica, siendo fieles a su naturaleza de responder oportunamente en momentos de crisis, también se ha visto envuelto en cambios que lo alejan cada día más de su escenario prinpical, el quirófano.

En el marco de la información recibida desde Asia y Europa ${ }^{\mathrm{I}-4}$, diversas organizaciones advirtieron sobre las medidas necesarias para mejorar la capacidad de respuesta de los sistemas de salud en el mundo ${ }^{5-7}$. Los Centros para el Control y la prevención de Enfermedades de los Estados Unidos (CDC, por sus siglas en ingles) establecen tres niveles operacionales para optimizar el uso de elementos de protección personal: convencional, contingencia y crisis. Estos niveles permiten direccionar las estrategias que van, desde el aplazamiento de la consuta externa y el retiro de suministro de tapabocas en sitios públicos, hasta la cancelación de todos los procedimientos programados no urgentes y el reuso de los elementos de protección personal ${ }^{8}$. Adicionalmente, la incorporación de herramientas digitales y la instalación de hospitales temporales han permitido responder eficientemente a la demanda asistencial durante la pandemia.

Existe poca información disponible sobre el impacto que generan los cambios adoptados en los servicios de cirugia en Colombia. El objetivo de la primera fase de este proyecto es describir la percepción colectiva de la comunidad quirúrgica sobre los efectos de la pandemia COVID-i9 en sus servicios, una vez fue decretada la fase de mitigación.

\section{Métodos}

\section{Estudio, población y diseño de la encuesta}

Estudio descriptivo de corte transversal tipo encuesta. Se tomó como población de estudio las personas que cumplieran con al menos uno de los siguienes criterios: ser médico residente de cirugía general de uno de los pro- 
gramas académicos en Colombia, ser médico especialista en Cirugía general con práctica clínica vigente en Colombia, o ser cirujano sub-especialista con práctica clínica vigente en Colombia. Mediante un método Delphi modificado ${ }^{9}$ se definieron los dominios a evaluar en una encuesta estructurada compuesta por Io preguntas de selección múltiple y 8 preguntas con respuesta tipo Likert.

\section{Recopilación y procesamiento de datos}

Utilizando el software disponible en el dominio público surveyplanet.com $(2)$ (2020 Survey Planet, LLC), se distribuyó la encuesta con muestreo por conveniencia, durante la fase de mitigación de la pandemia por COVID-I9 en Colombia. Se publicó en las plataformas de redes sociales Twitter y Facebook, y se contó con el apoyo de la Asociacion Colombiana de Cirugía para su distribución por medio de correo electrónico a todos los miembros de la misma.

Se permitió un periodo de actividad para responder la encuesta del 5 al 20 de Abril de 2020 y se restringió para ser tomada desde el mismo dispositivo electrónico solo una vez. Se agruparon las respuestas de los participantes provenientes de las áreas metropolitanas así: Bogota, Chía y Soacha, en área metropolitana de Bogota; Medellín, Itagüi, Envigado y Bello, en área metropolitana del Valle de Aburrá; Cali y Palmira en área metropolitana de Cali; y Bucaramanga, Floridablanca y Piedecuesta, en área metropolitana de Bucaramanga.

\section{Análisis estadístico}

Los resultados de la encuesta se exportaron y analizaron en Stata I5 (StataCorp LP, College Station, TX, USA). Los datos se describen como proporciones (en porcentaje) para variables categóricas y como medianas con rango intercuartil (RIQ) para variables continuas. Se utilizaron pruebas no paramétricas de $\mathrm{Chi}^{2}$ de Pearson, prueba exacta de Fisher o Kruskall-Wallis para comparar variables categóricas u ordinales, cuando fue apropiado. De igual manera, se usó la prueba V de Cramer como medida de tamaño de efecto entre variables categóricas. Todas las pruebas estadísticas fueron de 2 lados y el valor de $\mathrm{p}<0.05$ se consideró significativo.

\section{Resultados}

De los 477 encuestados, se excluyeron 13 participantes por no cumplir con los criterios de inclusión. Entre los 464 participantes restantes, la mediana de edad fue 38 años (RIQ 3I, 50.5), $27.8 \%(\mathrm{n}=129)$ fueron mujeres, y $45.3 \%(\mathrm{n}=2 \mathrm{IO})$ cirujanos generales. El hospital universitario privado (48.3\%, $\mathrm{n}=224$ ) fue el tipo de institución hospitalaria de mayor representación en la muestra (tabla I). Adicionalmente, la mayor parte de los participantes provinieron del area metropolitana de Bogotá (5I.4\%, $\mathrm{n}=239$ ), seguido por el area metropolitana del Valle de Aburra (II.6\%, n=54), y Area metropolitana de Cali (II.6\%, $\mathrm{n}=54)$.

El 2I.5\% (n=IOO) de los participantes reportó haber tenido contacto directo con paciente COVID-I9 positivo. Al estratificar la noción de contacto directo por nivel de entrenamiento, tipo de institución hospitalaria y ciudad, encontramos que la mayor proporción de participantes con noción de contacto con paciente COVID-I9 positivo trabajan en Bogota ( $66 \%$, p<0.0oI), sin diferencia significativa en las otras variables (tabla 2 ).

\section{Dominio recomendaciones de bioseguridad}

De acuerdo a las recomendaciones para procedimientos quirúrgicos en pacientes COVID-I9 positivos, el $78 \%(\mathrm{n}=366)$ de los encuestados consideraron que las medidas de protección son 'muy efectivas' ( $n=I 2 I)$ o 'efectivas' $(n=245)$. De igual manera, el $75.6 \%$ ( $\mathrm{n}=35 \mathrm{I})$ de los encuestados reportó sentirse 'muy seguro' o 'seguro' con estas recomendaciones para realizar procedimientos quirúrgicos. Al estratificar estas variables por nivel de entrenamiento, no hubo diferencia significativa $(p>0.05)$ en la percepción 
Tabla 1. Caracteristicas Demograficas de la población encuestada

\begin{tabular}{|c|c|}
\hline Variables Demográficas & $\mathrm{N}=464$ \\
\hline Edad en años, mediana (RIQ) & $38(31-50.5)$ \\
\hline Sexo, Femenino, n (\%) & $129(27.8 \%)$ \\
\hline \multicolumn{2}{|l|}{ Nivel de entrenamiento } \\
\hline Cirujano general & $210(45.3 \%)$ \\
\hline Cirujano sub-especialista & $141(30.4 \%)$ \\
\hline Médico residente & $113(24.4 \%)$ \\
\hline \multicolumn{2}{|l|}{ Ciudad } \\
\hline Área metropolitana de Bogotá & $239(51.4 \%)$ \\
\hline Área metropolitana del Valle de Aburra & $54(11.6 \%)$ \\
\hline Área metropolitana de Cali & $54(11.6 \%)$ \\
\hline Área metropolitana de Bucaramanga & $19(4.1 \%)$ \\
\hline Barranquilla & $13(2.8 \%)$ \\
\hline Cartagena & $16(3.4 \%)$ \\
\hline Manizales & $7(1.5 \%)$ \\
\hline Neiva & $7(1.5 \%)$ \\
\hline Armenia & $4(0.9 \%)$ \\
\hline Cúcuta & $4(0.9 \%)$ \\
\hline Ibagué & $4(0.9 \%)$ \\
\hline Popayán & $4(0.9 \%)$ \\
\hline Pasto & $4(0.9 \%)$ \\
\hline Pereira & $3(0.6 \%)$ \\
\hline Ubaté & $3(0.6 \%)$ \\
\hline Valledupar & $3(0.6 \%)$ \\
\hline Barrancabermeja & $2(0.4 \%)$ \\
\hline Girardot & $2(0.4 \%)$ \\
\hline Montería & $2(0.4 \%)$ \\
\hline Villavicencio & $2(0.4 \%)$ \\
\hline Aguachica & $1(0.2 \%)$ \\
\hline Apartado & $1(0.2 \%)$ \\
\hline Granada & $1(0.2 \%)$ \\
\hline Florencia & $1(0.2 \%)$ \\
\hline La Dorada & $1(0.2 \%)$ \\
\hline Magangué & $1(0.2 \%)$ \\
\hline Pamplona & $1(0.2 \%)$ \\
\hline Riohacha & $1(0.2 \%)$ \\
\hline Salamina & $1(0.2 \%)$ \\
\hline Sincelejo & $1(0.2 \%)$ \\
\hline Tuluá & $1(0.2 \%)$ \\
\hline Tunja & $1(0.2 \%)$ \\
\hline \multicolumn{2}{|l|}{ Lugar de trabajo } \\
\hline Privado Universitario & $224(48.3 \%)$ \\
\hline Público Universitario & $118(25.4 \%)$ \\
\hline Privado No Universitario & $100(21.6 \%)$ \\
\hline Público No Universitario & $22(4.7 \%)$ \\
\hline
\end{tabular}

de las recomendaciones de bioseguridad entre la comunidad quirúrgica (tabla 3 ).

A pesar de esto, el $63.7 \%(n=296)$ de los participantes reportaron que estas recomendaciones se 'cumplen parcialmente', mientras I7 $\%(n=80)$ considera que se 'cumplen completamente'. Las diferencias observadas no tuvieron significancia estadística entre médicos residentes y cirujanos (tabla 3). Al evaluar esta variable con respecto al tipo de institución hospitalaria, se observó diferencia en las respuestas entre hospiales públicos y privados ( $\mathrm{p}=\mathrm{0.004}$ ) (figura $\mathrm{I})$, con una tendencia a percibir que las recomendaciones 'no se cumplen' en los hospitales públicos $(22.9 \%$ vs. Io.8 \%) y que se 'cumplen completamente' en los hospitales privados (I8.0 \% vs. I3.6 \%). A pesar de la diferencia estadísticamene significativa, el tamaño del efecto del tipo hospitalario en esta apreciación fue leve (prueba de Cramer o.I6).

Tabla 2. Noción de contacto con paciente COVID-19 positivo

\begin{tabular}{|c|c|c|c|}
\hline Variables & $\underset{n=100}{S i}$ & $\begin{array}{c}\text { No } \\
n=364\end{array}$ & $\mathrm{p}$-valor \\
\hline $\begin{array}{l}\text { Nivel de } \\
\text { entrenamiento, } n(\%)\end{array}$ & & & 0.29 \\
\hline Cirujano General & $40(40.0 \%)$ & $170(46.7 \%)$ & \\
\hline $\begin{array}{l}\text { Cirujano Sub- } \\
\text { especialista }\end{array}$ & $30(30.0 \%)$ & $111(30.5 \%)$ & \\
\hline Residente & $30(30.0 \%)$ & $83(22.8 \%)$ & \\
\hline $\begin{array}{l}\text { Tipo de institución } \\
\text { hospitalaria, } \mathbf{n}(\%)\end{array}$ & & & 0.67 \\
\hline Privado Universitario & $53(53.0 \%)$ & $171(47.0 \%)$ & \\
\hline Público Universitario & $25(25.0 \%)$ & $93(25.5 \%)$ & \\
\hline $\begin{array}{l}\text { Privado No } \\
\text { Universitario }\end{array}$ & $19(19.0 \%)$ & $81(22.3 \%)$ & \\
\hline $\begin{array}{l}\text { Público No } \\
\text { Universitario }\end{array}$ & $3(3.0 \%)$ & $19(5.2 \%)$ & \\
\hline Ciudad, n (\%) & & & 0.001 \\
\hline Bogotá & $66(66.0 \%)$ & $173(47.5 \%)$ & \\
\hline Cali & $10(10.0 \%)$ & $43(11.8 \%)$ & \\
\hline Medellín & $7(7.0 \%)$ & $47(12.9 \%)$ & \\
\hline Neiva & $3(3 \%)$ & $4(1 \%)$ & \\
\hline Cartagena & $2(2.0 \%)$ & $14(3.8 \%)$ & \\
\hline Barraquilla & $2(2.0 \%)$ & $11(3.0 \%)$ & \\
\hline Pasto & $2(2.0 \%)$ & $2(0.5 \%)$ & \\
\hline Popayán & $2(2.0 \%)$ & $2(0.5 \%)$ & \\
\hline
\end{tabular}


Tabla 3. Percepción sobre recomendaciones de bioseguridad

\begin{tabular}{|c|c|c|c|c|c|}
\hline Pregunta & $\begin{array}{c}\text { Total } \\
(n=464)\end{array}$ & $\begin{array}{l}\text { Cirujano } \\
\text { General } \\
(\mathrm{n}=\mathbf{2 1 0})\end{array}$ & $\begin{array}{l}\text { Cirujano Sub- } \\
\text { especialista } \\
(n=141)\end{array}$ & $\begin{array}{c}\text { Médico } \\
\text { Residente } \\
(n=113)\end{array}$ & $P$ valor \\
\hline $\begin{array}{l}\text { Considera que las recomendaciones de bio- } \\
\text { seguridad durante procedimientos quirúrgicos } \\
\text { con pacientes COVID-19 positivo son: }\end{array}$ & & & & & 0.29 \\
\hline Muy efectivas o Efectivas & $366(78.8 \%)$ & $163(77.6 \%)$ & $119(84.4 \%)$ & $84(74.3 \%)$ & \\
\hline No sabe & $8(1.7 \%)$ & $5(2.4 \%)$ & $1(0.7 \%)$ & $2(1.8 \%)$ & \\
\hline Inefectivas o Sin evidencia & $90(19.4 \%)$ & $42(20.0 \%)$ & $21(14.9 \%)$ & $27(23.9 \%)$ & \\
\hline $\begin{array}{l}\text { Con respecto a las recomendaciones de bio- } \\
\text { seguridad durante procedimientos quirúrgicos } \\
\text { con pacientes COVID-19 positivo, usted se } \\
\text { siente: }\end{array}$ & & & & & 0.41 \\
\hline Muy seguro o Seguro & $351(75.6 \%)$ & $161(76.7 \%)$ & $110(78.0 \%)$ & $80(70.8 \%)$ & \\
\hline Indiferente & $4(0.8 \%)$ & $3(1.4 \%)$ & $0(0.0 \%)$ & $1(0.9 \%)$ & \\
\hline Muy inseguro o Inseguro & $109(23.4 \%)$ & $46(21.9 \%)$ & $31(22.0 \%)$ & $32(28.3 \%)$ & \\
\hline $\begin{array}{l}\text { Considera que las recomendaciones de bio- } \\
\text { seguridad durante los procedimientos quirúr- } \\
\text { gicos en paciente COVID-19 positivo, se están } \\
\text { cumpliendo: }\end{array}$ & & & & & 0.06 \\
\hline Completamente & $80(17.2 \%)$ & $34(16.2 \%)$ & $33(23.4 \%)$ & $13(11.5 \%)$ & \\
\hline Parcialmente & $296(63.7 \%)$ & $137(65.2 \%)$ & $88(62.4 \%)$ & $71(62.8 \%)$ & \\
\hline No se están cumpliendo & $67(14.4 \%)$ & $27(12.9 \%)$ & $17(12.1 \%)$ & $23(20.4 \%)$ & \\
\hline No sabe & $21(4.5 \%)$ & $12(5.7 \%)$ & $3(2.1 \%)$ & $6(5.3 \%)$ & \\
\hline $\begin{array}{l}\text { En el escenario de no contar con los elementos } \\
\text { de protección personal completos, esta dis- } \\
\text { puesto a realizar procedimientos quirúrgicos } \\
\text { urgentes en pacientes COVID19 positivos: }\end{array}$ & & & & & 0.04 \\
\hline $\mathrm{Si}$ & $50(10.8 \%)$ & $22(10.5 \%)$ & $9(6.4 \%)$ & $19(16.8 \%)$ & \\
\hline No & $356(76.9 \%)$ & $156(74.6 \%)$ & $115(81.6 \%)$ & $85(75.2 \%)$ & \\
\hline No sabe & $57(12.3 \%)$ & $31(14.8 \%)$ & $17(12.1 \%)$ & $9(8.0 \%)$ & \\
\hline
\end{tabular}
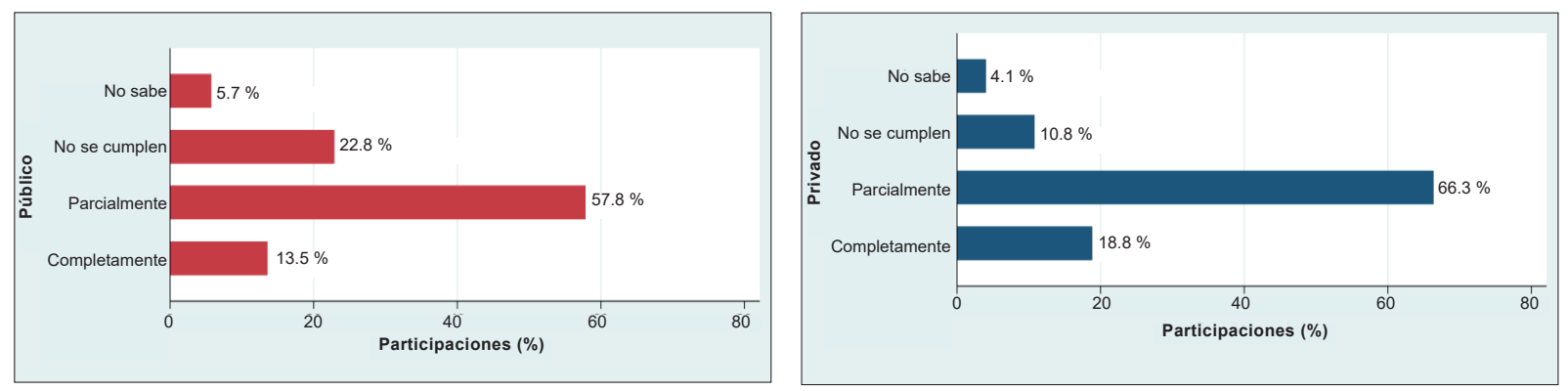

Figura 1. Percepción del cumplimiento de las recomendaciones de bioseguridad durante los procedimientos quirúrgicos según el tipo de institución hospitalaria 
Finalmente al preguntárseles si estarían dispuestos a realizar un procedimiento de urgencia en un paciente COVID-I9 positivo sin los elementos de protección personal completos, el $76.9 \%(n=356)$ de los encuestados consideraron 'no estar dispuesto' a tomar el riesgo, con una leve tendencia a 'sí estar dispuesto' entre los médicos en entrenamiento ( $\mathrm{p}=0.04$; Cramer o.I).

\section{Dominio percepción en los servicios de urgencias}

Con respecto al impacto de la pandemia sobre los patrones de consulta a los servicios de urgencias, se observó que el $73 \%(n=293)$ de los participantes consideraron que las urgencias secundarias al trauma se han disminuido en más del $50 \%$. Cabe resaltar que menos del $2 \%$ de los encuestados consideran que la pandemia no ha tenido ningún impacto en las consultas secundarias al trauma (tabla 4). En contraste, no se observó una distribución diferencial significativa en la percepción del impacto en las consultas secundarias a patologías inflamatorias, con $33 \%(\mathrm{n}=\mathrm{I} 55)$ de los participantes reportando un descenso entre el 25-50 \%. Sin embargo, la mayoría de los encuestados $(75 \%, \mathrm{n}=348)$ esperan que aumenten las complicaciones en patologías inflamatorias, debido a tiempos de evolución prolongados al momento de la consulta. Al evaluar la percepción de los patrones de consulta secundarias a

Tabla 4. Percepción sobre patrones de consulta al servicio de urgencias

\begin{tabular}{|c|c|c|c|c|c|}
\hline Pregunta & Total $(n=464)$ & $\begin{array}{l}\text { Cirujano } \\
\text { General } \\
(n=210)\end{array}$ & $\begin{array}{l}\text { Cirujano Sub- } \\
\text { especialista } \\
(n=141)\end{array}$ & $\begin{array}{l}\text { Médico } \\
\text { Residente } \\
(n=113)\end{array}$ & $\mathbf{P}$ valor \\
\hline $\begin{array}{l}\text { Considera que las consultas a urgencias } \\
\text { secundarias a trauma se han disminuido } \\
(n=401) \text { : }\end{array}$ & & & & & 0.004 \\
\hline$>75 \%$ & $180(45 \%)$ & $90(47.4 \%)$ & $58(54.7 \%)$ & $32(30.5 \%)$ & \\
\hline $50-75 \%$ & $113(28 \%)$ & $59(31.1 \%)$ & $18(17.0 \%)$ & $36(34.3 \%)$ & \\
\hline $25-50 \%$ & $76(19 \%)$ & $27(14.2 \%)$ & $24(22.6 \%)$ & $25(23.8 \%)$ & \\
\hline$<25 \%$ & $26(6.5 \%)$ & $11(5.8 \%)$ & $6(5.7 \%)$ & $9(8.6 \%)$ & \\
\hline No se han disminuido & $6(1.5 \%)$ & $3(1.6 \%)$ & 0 & $3(2.9 \%)$ & \\
\hline $\begin{array}{l}\text { Considera que las consultas a urgencias se- } \\
\text { cundarias a patologías inflamatorias se han } \\
\text { disminuido ( } n=422) \text { : }\end{array}$ & & & & & 0.64 \\
\hline$>75 \%$ & $48(10 \%)$ & $45(22.6 \%)$ & $13(11.7 \%)$ & $13(11.6 \%)$ & \\
\hline $50-75 \%$ & $108(23 \%)$ & $45(22.6 \%)$ & $36(32.4 \%)$ & $27(24.1 \%)$ & \\
\hline $25-50 \%$ & $155(33.4 \%)$ & $73(36.7 \%)$ & $39(35.1 \%)$ & $43(38.4 \%)$ & \\
\hline$<25 \%$ & $65(14 \%)$ & $34(17.1 \%)$ & $12(10.8 \%)$ & $19(17.0 \%)$ & \\
\hline No se han disminuido & $46(9.9 \%)$ & $25(12.6 \%)$ & $11(9.9 \%)$ & $10(8.9 \%)$ & \\
\hline $\begin{array}{l}\text { Considera que van a aumentar las complica- } \\
\text { ciones secundarias a patología inflamatoria } \\
\text { por tiempos de evolución prolongados antes } \\
\text { de la consulta? }\end{array}$ & & & & & 0.87 \\
\hline Totalmente de acuerdo o De acuerdo & $348(75.1 \%)$ & $158(75.6 \%)$ & $106(75.2 \%)$ & $84(74.3 \%)$ & \\
\hline Indiferente & $56(12.1 \%)$ & $22(10.5 \%)$ & $19(13.5 \%)$ & $15(13.3 \%)$ & \\
\hline $\begin{array}{l}\text { Totalmente en desacuerdo o } \\
\text { En desacuerdo }\end{array}$ & $59(12.7 \%)$ & $29(13.9 \%)$ & $16(11.3 \%)$ & $14(12.4 \%)$ & \\
\hline
\end{tabular}


Continuación

Tabla 4. Percepción sobre la capacidad de respuesta

\begin{tabular}{|c|c|c|c|c|c|}
\hline Pregunta & Total $(n=464)$ & $\begin{array}{l}\text { Cirujano } \\
\text { General } \\
(n=210)\end{array}$ & $\begin{array}{l}\text { Cirujano Sub- } \\
\text { especialista } \\
(n=141)\end{array}$ & $\begin{array}{l}\text { Médico } \\
\text { Residente } \\
(n=113)\end{array}$ & $P$ valor \\
\hline $\begin{array}{l}\text { Con respecto a la opción de cancelar todas las ciru- } \\
\text { gías oncológicas en su institución, usted considera } \\
\text { estar }\end{array}$ & & & & & 0.93 \\
\hline Totalmente de acuerdo o De acuerdo & $145(31.2 \%)$ & $69(32.9 \%)$ & $40(28.4 \%)$ & $36(31.9 \%)$ & \\
\hline Indiferente & $26(5.6 \%)$ & $12(5.7 \%)$ & $8(5.7 \%)$ & $6(5.3 \%)$ & \\
\hline Totalmente en desacuerdo o En desacuerdo & $293(63.1 \%)$ & $129(61.4 \%)$ & $93(66.0 \%)$ & $71(62.8 \%)$ & \\
\hline $\begin{array}{l}\text { Con respecto a la opción de cancelar todas las ciru- } \\
\text { gías electivas no esenciales en su institución, usted } \\
\text { considera estar }\end{array}$ & & & & & 0.74 \\
\hline Totalmente de acuerdo o De acuerdo & $430(93 \%)$ & $194(92.4 \%)$ & $129(91.5 \%)$ & $107(94.7 \%)$ & \\
\hline Indiferente & $6(1 \%)$ & $4(1.9 \%)$ & $1(0.7 \%)$ & $1(0.9 \%)$ & \\
\hline Totalmente en desacuerdo o En desacuerdo & $28(6 \%)$ & $12(5.7 \%)$ & $11(7.8 \%)$ & $5(4.4 \%)$ & \\
\hline $\begin{array}{l}\text { Con respecto a la opción de establecer la estrategia } \\
\text { de colecistostomía mas antibióticos como primera } \\
\text { línea en pacientes con colecistitis aguda en su } \\
\text { institución, usted considera estar }(n=443)\end{array}$ & & & & & 0.43 \\
\hline Totalmente de acuerdo o De acuerdo & $189(42.6 \%)$ & $87(44.2 \%)$ & $52(38.5 \%)$ & $50(45.0 \%)$ & \\
\hline Indiferente & $16(3.6 \%)$ & $6(3.0 \%)$ & $8(5.9 \%)$ & $2(1.8 \%)$ & \\
\hline Totalmente en desacuerdo o En desacuerdo & $238(53.7 \%)$ & $104(52.8 \%)$ & $75(55.6 \%)$ & $59(53.2 \%)$ & \\
\hline $\begin{array}{l}\text { ¿Se considera usted en capacidad de brindar soporte } \\
\text { ventilatorio mecánico, en caso de ser necesario? }\end{array}$ & & & & & 0.48 \\
\hline $\mathrm{Si}$ & $209(45 \%)$ & $101(48.1 \%)$ & $59(41.8 \%)$ & $49(43.4 \%)$ & \\
\hline No & $255(55 \%)$ & $109(51.9 \%)$ & $82(58.2 \%)$ & $64(56.6 \%)$ & \\
\hline
\end{tabular}

trauma y patología inflamatoria por tipo de hospital, no se observaron diferencias significativas entre hospitales públicos y privados ( $\mathrm{p}=$ =.I y 0.4 , respectivamente).

\section{Dominio percepción de la capacidad de respuesta y adaptación}

El $46 \%$ (n=2I4) de los encuestados reportó 'estar dispuesto' a ser segunda línea de atención en el servicio hospitalario que sea necesario (figura 2). A pesar de que solo el $13.3 \%(n=62)$ de los encuestados reportaron 'estar dispuesto' a apoyar en el servicio de cuidado intensivo, el $45 \%(\mathrm{n}=209)$ de los participantes se consideran en la capacidad de dar soporte ventilatorio mecánico. Adicionalmente, no se observaron diferencias significativas en las preferencias de los servicios, ni en la capacidad reportada para brindar soporte ventilatorio mecánico, según el nivel de entrenamiento quirúrgico $(\mathrm{p}=0.09$ y 0.48 , respectivamente).

Con respecto a las adaptaciones de los servicios quirúrgicos ambulatorios, el $93 \%(\mathrm{n}=430)$ de los participantes estuvo 'totalmente de acuerdo' o 'de acuerdo' en la cancelación de cirugías electivas no esenciales. Sin embargo, con respecto a los procedimientos electivos en pacientes oncológicos, aunque el $53.6 \%(n=249)$ consideró estar 'en desacuerdo' en cancelar cirugías oncológicas, una cuarta parte de los encuestados ( $25 \%, \mathrm{n}=\mathrm{II} 9)$ reportó estar 'de acuerdo' con esta medida (figura 3). No se observaron diferencias al comparar 


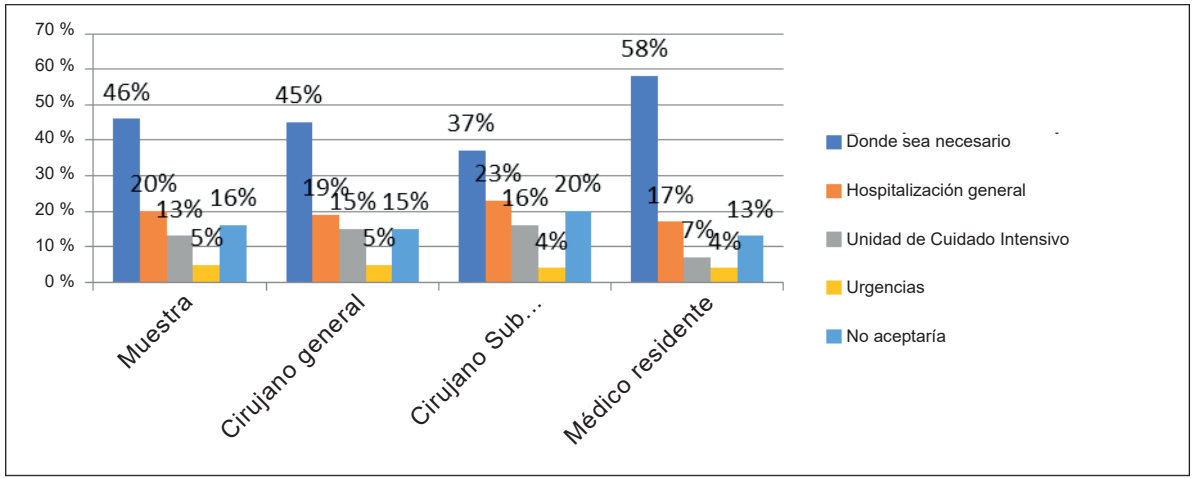

Figura 2. Distribución de las preferencias para prestar servicio en sitios de apoyo como segunda línea
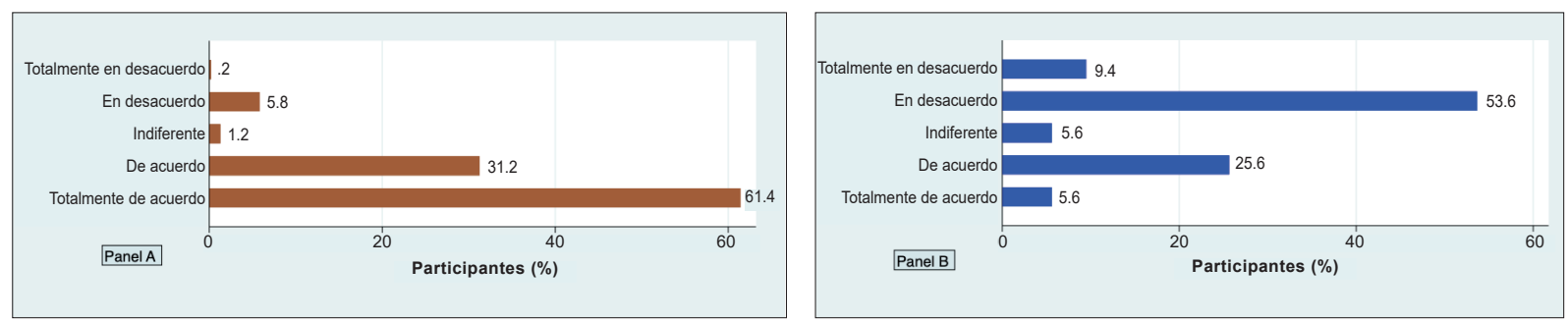

Figura 3. Distribución de la percepción de la comunidad quirúrgica con la cancelación de procedimientos quirúrgicos. Panel A corresponde a procedimientos electivos no esenciales. Panel B corresponde a procedimientos electivos oncológicos.

estas variables según el nivel de entrenamiento ni el tipo de hospital ( $>0.05)$.

\section{Discusión}

La pandemia por COVID-ı9 ha obligando a los diferentes actores a adaptarse para poder suplir las demandas en la atención en salud. Es así como los servicios quirúrgicos han tenido que modificar sus estrategias operacionales para estar en sintonía con esta coyuntura. Durante la fase de recolección de datos, el Ministerio de Salud reportó desde el 5 al 22 de abril, un aumento de 1065 a 4356 casos confirmados, mismo periodo en que $20 \%$ de los encuestados reportó haber tenido contacto directo con pacientes COVID-I9 positivo.

Los datos de esta encuesta muestran la percepción sobre el impacto que ha tenido la pandemia en los servicios de cirugía del país, indicando además hasta el momento, que existe voluntad de adaptación por parte de la comunidad quirúrgica .

En primer lugar, la percepción sobre la efectividad y seguridad de las recomendaciones de bioseguridad entre la comunidad quirúrgica es buena, sin una variabilidad significativa según el nivel de entrenamiento ni el lugar de trabajo. El Centro de Medicina Basada en la Evidencia de la Universidad de Oxford establece que aunque no exiten estudios directos con SARS-CoV-2 entre tapabocas quirúrgicos estandares y mascarillas de alta eficiencia tipo N95, FFP2 o FFP3, estas últimas deben ser utilizadas durante procedimientos médicos generadores de aerosoles (PMGA), según evidencia derivada en otras enfermedades virales respiratorias ${ }^{\text {Io }}$. Se recomienda además protección para los ojos, guantes y bata de manga larga, con una buena técnica de colocación y retirada. 
Aunque los datos sobre la afectación de los trabajadores de la salud en esta pandemia y otros brotes epidémicos demuestran el mayor riesgo que tenemos como médicos ${ }^{\mathrm{II}, 12}$, los hallazgos de este estudio son fundamentales para el funcionamiento de los servicios de cirugía, ya que al percibir las recomenadaciones como efectivas y seguras, los cirujanos y médicos residentes realizan sus labores con menor preocupación de infectarse.

Llama la atención que la mayoría de encuestados (63\%) perciben que estas recomendaciones se cumplen parcialmente, con una tendencia a considerar que existe una menor adherencia en los hospitales públicos que en los privados. Aunque el objetivo de esta encuesta no fue la medición directa de la adherencia a los protocolos de bioseguridad, varios factores han sido identificados por parte de los trabajadores de la salud, como barreras y facilitadores para cumplir con las recomendaciones de control y prevención de enfermedades infecciosas, entre ellos, el apoyo administrativo, la disponibilidad del espacio físico para aislamiento, el entrenamiento y el acceso a los elementos de protección personal ${ }^{13}$.

Sin embargo, la percepción de falta de elementos de protección personal es un fenómeno recurrente en varios paises del mundo ${ }^{14,15}$. El Colegio Médico de Chile encuestó a 2.227 trabajadores de la salud del área pública y privada, encontrando que el $85.7 \%$ de los encuestados reportaba falta de alguno de los implementos de bioseguridad ${ }^{16}$. Igualmente, la Federación Médica Colombiana encuestó a 939 trabajadores de la salud, y $87.7 \%$ reportaron carencia de máscaras $\mathrm{N} 95{ }^{17}$.

Los datos también muestran cómo la comunidad quirúrgica percibe de forma homogénea una disminución significativa en las consultas en los servicios de urgencias secundarias al trauma. Estos datos se corresponden con los resultados de un Centro de Trauma nivel I en Nueva Zelanda, en donde reportaron una disminución del $43 \%$ en todas las admisiones relacionadas con trauma, y hasta del $50 \%$ de aquellas clasificadas como trauma severo durante la fase de confinamiento
${ }^{18}$. En conrtraste, Hatchimonji y colaboradores ${ }^{19}$ reportaron un aumento de las lesiones por proyectiles de arma de fuego en la ciudad de Filadelfia durante el mes de Marzo, con 52 heridos durante los primeros io dias de establecerse la orden de confinamiento. Aunque la percepción en nuestros servicios de urgencias respecto al trauma es buena hasta el momento, esta claro que este comportamiento esta relacionado, tanto con la severidad de las restricciones impuestas, como con la presencia de otros factores sociodemográficos en cada comunidad.

Por otro lado, la disminución en las consultas a urgencias secundarias a patologías inflamatorias no fue percibida de forma generalizada, pues se prevee que los procesos de enfermedad son independientes de las estrategias de confinamiento, del mismo modo como los patrones de consulta se ven influenciados por la percepción del riesgo de infección por parte de la población general. Algunos centros en Estados Unidos han reportado una disminución en las consultas a los servicios de urgencias, al igual que el número de muertes secundario a eventos cardiovasculares y cerebrovasculares ${ }^{20,21}$. Es igualmente llamativo que los encuestados consideran probable el aumento de las complicaciones, debido a tiempos de evolución prolongados al momento de acudir a los servicios de urgencias, lo cual podría esperarse en los centros en donde ya se ha percibido una disminución en la consultas durante la primera mitad del mes de Abril. Será de gran interés observar el comporamiento de estos datos a medida que avance la pandemia.

Finalmente, se observó que los encuestados estan de acuerdo con las estrategias adoptadas dentro de las instituciones hospitalarias, en respuesta a lo que se prevee será un aumento del uso de recursos institucionales, incluso cuando la cancelación de cirugías electivas y el aumento del riesgo de infección implican sacrificios individuales, tanto para cirujanos como para médicos residentes. La crisis vivida en el norte de Italia hizo que los servicios de cirugía en el mundo enfocaran los recursos disponibles de personal médico y salas de cirugía para mejorar la capacidad 
de respuesta ${ }^{22}$. Adicionalmente, series de casos indican hasta $20 \%$ de complicaciones postoperatorias por SARS-CoV-2 en pacientes llevados a cirugía durante el periodo de incubación ${ }^{23}$.

Observamos que $73 \%$ de los participantes reportó estar dispuesto a ser reubicado en otros servicios, y que $93 \%$ estuvo de acuerdo con la cancelación de cirugías electivas no esenciales. Aunque para el momento de la realización del estudio los casos de COVID-I9 confirmados en Colombia no alcanzan la fase de crisis, la mayoría de los servicios quirúrgicos habían cancelado los programas de cirugía electiva, a la espera de observar el comportamiento de la curva de contagio.

Por otro lado, aunque series en pacientes oncológicos indican mayor riesgo de infección ${ }^{24,25}$, la mayoría de los encuestados reportó estar en desacuerdo con cancelar cirugías electivas oncológicas. El Colegio Americano de Cirujanos (ACS, por sus siglas en ingles) ${ }^{26}$, recomendó realizar una evaluación individual de los pacientes oncológicos según la estabilidad de la enfermedad, la fase de la pandemia y la disponibilidad de recursos de los hospitales locales. Según los datos de la encuesta, para el momento de la pandemia en que nos encontramos en Colombia, se puede esperar que aun se conciba la realización de procedimientos quirúrgicos electivos en pacientes oncológicos.

\section{Limitaciones}

Este trabajo tiene las limitaciones inherentes a los estudios con encuestas en la medida que simplifican la realidad y pueden conducir a conclusiones sesgadas, especialmente con el uso de preguntas de selección múltiple. Adicionalmente, existe un sesgo de selección ya que hubo mayor representación de participantes provenientes de las principales ciudades y de hospitales privados.

\section{Conclusiones}

Para el inicio de la fase de mitigación en Colombia existe un consenso en la percepción del impacto de la pandemia en los servicios de cirugía entre cirujanos y médicos residentes, quienes reportaron haber tenido contacto directo con pacientes COVID-I9 positivos en $20 \%$ de los participantes. No se observó una variabilidad significativa en esta percepción respecto al nivel de entrenamiento ni al tipo institución hospitalaria. Adicionalmente, a pesar de que las recomendaciones de bioseguridad son consideradas efectivas y seguras por parte de médicos residentes y cirujanos, hubo una tendencia entre la mayoría de los encuestados a considerar que la adherencia a estas recomendaciones es parcial. Debido a la naturaleza de los estudios de corte transversal, es necesario evaluar la impacto de la pandemia según sú evolucion en el tiempo.

\section{Agradecimientos}

Agradecemos a la Asociacion Colombiana de Cirugia, en cabeza de la doctora Lilian Torregrosa y el doctor Oscar Guevara, por el apoyo y las recomendaciones durante la elaboración de este proyecto.

\section{Cumplimiento de normas éticas}

Consentimiento informado: Se considera que el estudio no conlleva problemas éticos dado que se asegura la confidencialidad de la información, se trata de datos anónimos, la encuesta es voluntaria y no se comparte información confidencial de pacientes o instituciones, por lo que no se requiere consentimiento informado ni autorización del Comité de Ética de las instituciones.

Declaración de conflicto de intereses: Los autores no declararon ningún conflicto de interés. Fuentes de financiación: Recursos propios de los autores.

\section{Referencias}

I. Ahmed S, Tan WLG, Chong YL. Surgical response to COVID-I9 pandemic: A Singapore perspective. J Am Coll Surg. 2020 Apr; Article in press.

https://doi.org/I0.IOI6/j.jamcollsurg.2020.04.003

2. Livingston E, Bucher K. Coronavirus disease 2019 (COVID-I9) in Italy. JAMA. 2020 Mar 17. Online ahead of print. https://doi.org/IO.IOoI/jama.2020.4344. 
3. Legido-Quigley H, Mateos-García JT, Campos VR, Gea-Sánchez M, Muntaner C, McKee M. The resilience of the Spanish health system against the COVID-I9 pandemic. Lancet Public Health. 2020 Mar I8. Online ahead of print.

https://doi.org/IO.IOI6/S2468-2667(20)30060-8.

4. Zu ZY, Jiang M Di, Xu PP, Chen W, Ni QQ, Lu GM, et al. Coronavirus disease 2019 (COVID-I9): A perspective from China. Radiology. 2020 Feb 2I;200490. Online ahead of print. https://doi.org/IO.II48/radiol.2020200490.

5. Ranney ML, Griffeth V, Jha AK. Critical supply shortages - The need for ventilators and personal protective equipment during the Covid-I9 pandemic. N Engl J Med. 2020 Apr 30;382(I8):e4I. Epub 2020 Mar 25. https://doi.org/Io.I056/NEJMp2006I4I.

6. Brindle M, Gawande A. Managing COVID-I9 in surgical systems. Ann Surg. 2020 Mar 23;IO.IO97/ SLA.0oooooooooo03923. Online ahead of print. https://doi.org/I0.I097/SLA.0000000000003923.

7. Torregrosa-Almonacid L, Prieto-Ortiz RG, Cabrera-Vargas LF, Ordoñez-Mosquera JM, Sánchez Cortés EF, Rodríguez-Vargas CM, et al. Recomendaciones generales para los Servicios de Cirugía en Colombia durante la pandemia COVID-I9 (SARS-CoV-2). Rev Colomb Cirugía [Internet]. 2020 Apr I6;35(2):265-8I. Disponible en: https://www.revistacirugia.org/index.php/cirugia/ article/view/633

8. Centers for Disease Control and Prevention. Interim infection prevention and control recommendations for patients with suspected or confirmed coronavirus disease (COVID-19) in healthcare settings. Fecha de consulta: 2020 Abril 26. Disponible en: https://www. cdc.gov/coronavirus/20I9-ncov/infection-control/control-recommendations.html

9. Carreño-Jaimes M. El método Delphi: cuando dos cabezas piensan más que una en el desarrollo de guías de práctica clínica. Rev Colomb Psiquiatr. 2009;38:185-93. Disponible en: http://www.scielo.org.co/scielo.php?script=sci_arttext\&pid=So034-74502009000I000I3\&ln$\mathrm{g}=\mathrm{en}$.

Io. Greenhalgh T, Chan XH, Khunti K, Durand-Moreau Q, Straube S, Devane D, et al. What is the efficacy of standard face masks compared to respirator masks in preventing COVID-type respiratory illnesses in primary care staff?. Oxford COVID-I9 Evidence Service. 2020 Mar 30. Disponible en: https://www.cebm.net/ wp-content/uploads/2020/03/COVID-CAT-PPE-MASKS-9-REVISED-002.pdf

II. Fowler RA, Lapinsky SE, Hallett D, Detsky AS, Sibbald WJ, Slutsky AS, et al. Critically ill patients with Severe Acute Respiratory Syndrome. JAMA. 2003;290:367-73. https://doi.org/Io.IooI/jama.290.3.367.
I2. Xiang YT, Jin Y, Wang Y, Zhang Q, Zhang L, Cheung T. Tribute to health workers in China: A group of respectable population during the outbreak of the COVID-I9. Int J Biol Sci. 2020;16:I739-40. https://doi.org/I0.7150/ ijbs.45I35.

I3. Houghton C, Meskell P, Delaney H, Smalle M, Glenton C, Booth A, et al. Barriers and facilitators to healthcare workers' adherence with infection prevention and control (IPC) guidelines for respiratory infectious diseases: a rapid qualitative evidence synthesis. Cochrane Database Syst Rev. 2020 Apr 2I. https://doi. org/IO.IOO2/I465I858.CDoI3582.

I4. Rimmer A. Covid-I9: Third of surgeons do not have adequate PPE, Royal College warns. BMJ. 2020 Apr I4;369:mI492. https://doi.org/Io.II36/bmj.mI492.

15. Ng K, Poon BH, Kiat Puar TH, Shan Quah JL, Loh WJ, Wong YJ, et al. COVID-I9 and the risk to health care workers: A case report. Ann Intern Med. 2020 Mar I6;L2O-OI75. Online ahead of print. https://doi. org/I0.7326/L2O-OI75.

I6. Colegio Médico de Chile A.G. Catastro de Elementos para Protección Personal. 2020. Disponible en: http:// www.colegiomedico.cl/catastro-epp/

17. Federación Médica Colombiana. Gremios médicos realizan encuesta del estado actual de bioseguridad y protocolos de atención COVID-I9. Fecha de consulta: 2020 Abril 26. Disponible en: https://www. federacionmedicacolombiana.com/2020/04/08/ gremios-medicos-realizan-encuesta-del-estado-actual-de-bioseguridad-y-protocolos-de-atencion-covid-I9/

I8. Christey G, Amey J, Campbell A, Smith A. Variation in volumes and characteristics of trauma patients admitted to a level one trauma centre during national level 4 lockdown for COVID-I9 in New Zealand. N Z Med J. 2020;24:8I-8.

I9. Hatchimonji JS, Swendiman RA, Seamon MJ, Nance ML. Trauma does not quarantine: Violence during the Covid-I9 pandemic. Ann Surg [Internet]. 2020; Disponible en: https://journals.lww.com/annalsofsurgery/ Documents/Trauma Does Not Quarantine.pdf

20. Appleby J. What is happening to non-covid deaths? BMJ. 2020 Apr 24;369:mI607. https://doi.org/Io.II36/bmj. mi607.

2I. Stradling R. Fewer people are going to Triangle emergency rooms, and that could be a bad thing. The News \& Observer. 2020. Fecha de consulta: 2020 Abril 26. Disponible en: https://www.newsobserver.com/news/ local/article242133886.html

22. Kurihara H, Bisagni P, Faccincani R, Zago M. COVID-I9 outbreak in northern Italy: Viewpoint of the Milan area surgical community. J Trauma Acute Care Surg. 2020 
Apr 3. Online ahead of print. https://doi.org/IO.IO97/ TA.0000000000002695.

23. Lei S, Jiang F, Su W, Chen C, Chen J, Mei W, et al. Clinical characteristics and outcomes of patients undergoing surgeries during the incubation period of COVID-I9 infection. EClinicalMedicine. $2020 \mathrm{Apr}$ 5;I0033I. Online ahead of print. https://doi.org/IO.IOI6/j. eclinm.2020.I0033I.

24. Dai M, Liu D, Liu M, Zhou F, Li G, Chen Z, et al. Patients with cancer appear more vulnerable to SARS-COV-2: a multi-center study during the COVID-I9 outbreak. Cancer Discov [Internet]. 2020 Jan I;CD-20-0422. Dis- ponible en: http://cancerdiscovery.aacrjournals.org/ content/early/2020/04/24/2159-8290.CD-20-0422.abstract

25. Burki TK. Cancer guidelines during the COVID-I9 pandemic. Lancet Oncol. 2020 Apr 2;SI470-2045(20)302I7-5. Online ahead of print. https://doi.org/IO.IOI6/SI4702045(20)30217-5.

26. American College of Surgeons. COVID-19 Guidelines for Triage of Cancer Surgery Patients. Online March 24, 2020. Fecha de consulta: 2020 Abril 26. Disponible en: https://www.facs.org/covid-rg/clinical-guidance/ elective-case/cancer-surgery. 